How to reference this article Majdzik Papić, K. (2020). Da Internet alla galleria d'arte, ovvero gli esperimenti traduttivi con gli emoji. Italica Wratislaviensia, 11(1), 169-190.

DOI: http://dx.doi.org/10.15804/IW.2020.11.1.07

Katarzyna Majdzik Papić

Uniwersytet Śląski w Katowicach, Polonia

katarzyna.majdzik@us.edu.pl

ORCID: 0000-0002-0532-4587

\title{
DA INTERNET ALLA GALLERIA D'ARTE, OVVERO GLI ESPERIMENTI TRADUTTIVI CON GLI EMOJI
}

\author{
FROM THE INTERNET TO THE ART GALLERY: \\ OR, ON EXPERIMENTS WITH EMOJIS \\ IN TRANSLATION
}

\begin{abstract}
The article presents various forms of intersemiotic and intrasemiotic translations which use emoji signs, i.e. iconic characters employed in digital communication by users of popular Internet applications and platforms (e.g. Twitter, Facebook and Messenger). The use of emojis is becoming more and more widespread, as evidenced by the increasing number of text messages and works of art which consist exclusively of these characters. At the same time, attempts are being made to translate more extensive verbal texts (including literary works) into the nonverbal system of emojis. One of the feats such efforts have so far accomplished is an experimental translation of Carlo Collodi's Pinocchio (La storia di un burattino), a classic of children's literature, from Italian to emojitaliano (a system codified by the participants of the experiment), which was developed on Twitter and appeared in print in 2017. Emoji-based works of art have also found their way to art galleries. The Garden of Emoji Delights, a triptych by Carla Gannis, is a conspicuous example of intrasemiotic translation (from a non-verbal code to another non-verbal code) involving Hieronymus Bosch's celebrated painting. Such applications of emoji are associated with paradigmatic changes in the humanities, which are related to the digital, iconic and performative turns and to today's remix culture (Lawrence Lessig, Lev Manovich).
\end{abstract}

Keywords: emoji, emojitaliano, experimental translation, digital translation, remix culture 


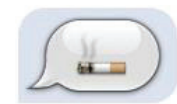

Ceci n'est pas une pipe ${ }^{1}$

\section{INTRODUZIONE}

T 9 immagine, icona digitale, che rappresenta una sigaretta accesa e l'iscrizione (titolo) Ceci n'est pas une pipe è la spiritosa creazione (emojipoem) di un anonimo composta da alcune parole e un'immagine: un segno emoji $i^{2}$. Un messaggio apparentemente insignificante, ovviamente rifacentesi alla celebre tela di René Magritte del 1929 intitolata La trahison des images (conosciuta anche come Ceci n'est pas une pipe), metaforicamente raccoglie in sé un insieme di problematiche riguardanti la natura della (ri/rap)presentazione nelle arti e, in generale, l'idea del simulacro, il rapporto della parola con l'immagine e l'immaginazione, la natura della creatività e della riproduzione, la relazione tra l'originale e la copia, tra la realtà virtuale e la fantasia, la lingua e la realtà. La battuta sta qui anche nel fatto che accanto al gioco intertestuale con l'originale (molte volte sottoposto ad analisi interpretative; cf. p. es. Foucault, 1973) abbiamo a che fare con un adattamento elettronico: l'immagine di una pipa sostituita da una sigaretta (è, quindi, una sorta di ricodifica e di conseguenza una traduzione in emoji), di fatto poco somigliante a quell'altro strumento di gran lunga più nobile (verrebbe da dire "analogico"). A prescindere dalla menzionata serie di problematiche (legate all'atto di minare la mimesi compiuto da Magritte

1 Tratto da Emojipoems (2012).

2 Gli emoji sono gli ideogrammi utilizzati dagli utenti di internet e delle applicazioni mobili nel processo di comunicazione elettronica. Rappresentano varie immagini di viso, oggetti, luoghi, tipi di tempo meteorologico, piante, animali, segnali di avvertimento (per esempio quelli stradali) ecc. Il loro aspetto e la quantità a disposizione degli utenti sono leggermente diversi a seconda dell'applicazione in questione o del sistema operativo (cf. Full Emoji List, v13.0 disponibile sulla pagina Unicode Consortium e i siti internet come emojipedia.org), tuttavia fanno parte dell'Unicode. Shigetaka Kurita è considerato progettista degli emoji (Prisco, 2018). 
e ripetuto dall'anonimo autore) concentrate in quest'opera parodico-dadaista, vale la pena di sottolineare il fatto che essa sia un'interpretazione abbastanza chiara della semipoetica digitale, la quale tradisce la tendenza a coinvolgere contemporaneamente varie forme del conoscere (per esempio linguistico, figurativo, acustico) e del percepire (cf. Szczęsna, 2018, pp. 132-133).

Notiamo inoltre che, benché esistano opere-emoji (cioè sequenze di segni emoji) composte esclusivamente da immagini, esse richiedono spesso (nonostante questo, immagino che sia comunque possibile un'esistenza autonoma di un tale genere di espressione) l'uso di testi verbali nel processo di interpretazione. Per uso di testi verbali intendo le situazioni in cui l'elemento iconico si intreccia con emoji e titolo o anche quando si alternano lettere/parole e immagini come succede nei rebus oppure quando l'immagine è letta come testo ${ }^{3}$. Il rifacimento dell'opera di Magritte ricorda le aporie che accompagnano non solo la svolta iconica (da tempo identificata nella cultura contemporanea), ma anche altri fenomeni artistici correlati nei quali l'elemento verbale è strettamente legato all'elemento iconico (per fare un esempio simile nel campo della liberatura: il rebus, da quello classico a quello incorporante emoji, o più in generale la scrittura visuale ${ }^{4}$ ). La compresenza di questi due ordini può essere vista come fonte di speranza (ricchezza interpretativa) o di minaccia (vicolo cieco dell'interpretazione):

[...] i due ordini possono essere in competizione tra di loro, o possono anche cooperare restituendo una diversa esperienza della lettura. In altre parole, la simultaneità dell'ibrido può assumere due vettori: può costruire una comunione teleologica dei due ordini o dei due mezzi semiotici (iconico e simbolico), d'altra parte può produrre un effetto diametralmente opposto: potrebbe

3 La piattaforma emojipoems.tumblr.com raccoglie numerosi esempi di opere che si avvalgono dei segni emoji.

4 Cf. tra gli altri Mitchell (2012); al margine bisogna aggiungere che la tela di Magritte (in una delle versioni in cui è rappresentato il riflesso dell'immagine della pipa) è un esempio di metaquadro (metapicture) secondo la classificazione di Mitchell (cf. anche Kalaga, 2010). 
portare alla dispersione dei sensi nell'esperienza di ricezione. (Kalaga, 2010, p. 19 ; trad. mia) $)^{5}$

Ad ogni modo, la compresenza di tipi di creatività multipli è caratteristica fondamentale della creazione artistica elettronica (soprattutto quando essa si spinge nel campo della sperimentazione) poiché i media elettronici sono dominati dalla onnicomprensiva estetica del collage.

Bożena Tokarz, studiosa polacca della traduzione artistica, paragona la situazione della traduzione con il rapporto degli oggetti con la loro rappresentazione nella seconda versione del quadro di Magritte Ceci n'est pas une pipe. Sulla tela vediamo un'immagine realistica di una pipa che sembra sospesa nell'aria. Sotto vi è un cavalletto con la tela raffigurante una (seconda) pipa e l'iscrizione Ceci n'est pas une pipe:

La rappresentazione realistica di entrambe le pipe porta alla seguente constatazione: l'imitazione è possibile, anche se falsa, infatti la pipa più grande è il prototipo concettuale e quella più piccola è la sua imitazione, come annuncia l'iscrizione. Tuttavia, ci sono più imitazioni perché anche la pipa più grande esiste solo nel mondo dell'immaginazione. Il quadro perciò esprime la situazione dell'arte, in particolare il suo status ontologico. [...] Entrambi i quadri di Magritte [anche La reproduction interdite] possono rappresentare una metafora della traduzione artistica. (Tokarz, 2010, p. 260; trad. mia)

Il rapporto della presentazione iconica della pipa con l'iscrizione del quadro è analogo alla relazione tra l'originale e la traduzione (cf. Tokarz, 1998, pp. 8-9). Il rifacimento elettronico dell'opera di Magritte, moltiplicando le rappresentazioni e trasportandole nel mondo virtuale, ci introduce invece nell'ambito delle considerazioni sulla traduzione digitale, sul ruolo della tecnica del collage nel processo traduttivo, sulla questione della soggettività nell'epoca tecnologizzata dei nuovi mezzi di comunicazione di massa. A questi problemi dedicheremo qualche parola presentando alcuni esempi di traduzione con l'uso di emoji.

${ }^{5}$ Questo problema nel contesto della traduzione nel sistema dei segni emoji è discusso in Majdzik (2018). 


\section{TRADUZIONE ED EMOJI}

Dal febbraio del 2016 sulla piattaforma Twitter si svolge il lavoro di traduzione della celebre opera di Carlo Collodi, Avventure di Pinocchio. Storia di un burattino, nella lingua dei segni digitali emoji. Francesca Chiusaroli (Università di Macerata) pubblica ogni giorno un tweet con un frammento del romanzo e gli utenti della piattaforma (e i coautori del progetto del "dizionario") cercano di proporre contenuti equivalenti utilizzando gli emoji. A fine giornata vengono scelte le soluzioni ritenute più valide e così va costruendosi il nuovo codice. Il processo di traduzione su Twitter è collegato al dizionario e al traduttore elettronico sulla piattaforma Telegram (@emojitalianobot) gestiti da Johanna Monti (Università di Napoli “L'Orientale") e da Federico Sangati (ricercatore indipendente, precedentemente legato - tra le altre - all'Università di Edimburgo). Nel 2017 la casa editrice Apice libri pubblica i risultati del gruppo di traduzione guidato dai linguisti italiani (Chiusaroli, 2017, pp. 5-18).

Bisogna sottolineare che questa traduzione è sorta come progetto scientifico nel corso del quale sono state elaborate rigorose regole grammaticali e un dizionario del cosiddetto emojitaliano. La versione cartacea contiene d'altronde entrambe le componenti (la grammatica e il dizionario) e la traduzione è presentata simultaneamente all'originale (l'originale e l'equivalente emoji sono stati posti l'uno accanto all'altro in base ai principi delle tavole sinottiche). I linguisti italiani sono stati i primi a produrre una così sistematica "lingua" emoji, l'idea della quale partiva dal presupposto di universalità, simile alla scrittura pittografica. È necessario altresì ricordare che i coordinatori si richiamavano a ispirazioni di modelli storici e di progetti di lingue artificiali benché il loro progetto sia anche un esperimento di riscrittura (re-writing) creativa (ivi, pp. 7-9). Tuttavia, la contraddizione primaria tra intento ed effetto è contenuta nel nome stesso del codice, emojitaliano, che ci rimanda a una lingua specifica, l'italiano. Nel lavoro sono state coinvolte numerose persone, utenti di Twitter, che - avvalendosi dei simboli presenti sulla tastiera degli emoji - assegnavano significati nuovi alle icone scelte o alle loro combinazioni. Questo lavoro ricordava pertanto la pro- 
duzione di un collage e consisteva nella giustapposizione di immagini o nella loro risemantizzazione (precisazione del significato e creazione di una voce nel dizionario).

I creatori del codice (partecipanti all'esperimento) nel corso del lavoro hanno sfruttato il potenziale iconico, indicale e simbolico dei segni emoji in quanto referenziali e astratti (laddove esprimono un concetto) cercando di evitare istanze di logografia (una siffatta creazione sarebbe stata di fatto universale) ${ }^{6}$. Non sempre però ciò è stato possibile, per esempio l'emoji che indica il numero 1, uno, sta a significare in Pinocchio l'articolo indeterminativo: un, un', una, uno. Un'incongruenza così piccola in una certa misura mette in questione la dimensione universale del codice prodotto. La lingua visuale degli emoji include comunque una componente verbale. La struttura dell'emojitaliano è basata sulle caratteristiche tipiche delle lingue naturali (per esempio nel campo dell'ordine delle "parole"/segni o nelle categorie grammaticali equivalenti) e poi i rari casi di logografia tradiscono la componente verbale del codice. Nel progetto sotto esame il problema traduttivo di base era rappresentato dal bisogno di trovare (creare) equivalenti pittografici delle unità lessicali italiane. I significati per i quali era facile trovare rappresentazioni dirette sono stati semplicemente sostituiti dai segni corrispondenti presenti sulla tastiera emoji, per esempio casa è indicata dall'icona della casa (immagine visuale prototipica di un piccolo edificio col tetto a spiovente). I segni verbali senza diretti equivalenti emoji sono restituiti adoperando segni composti, per esempio bottega nel codice emoji è una casa e degli attrezzi. Nella traduzione di alcuni concetti astratti ci si è serviti dell'immaginazione collettiva e delle tradizioni comuni. Così il lessema colpa è indicato da tre immagini: un uomo, una donna e una mela (ovvio riferimento biblico). Nella traduzione dei nomi propri con un significato preciso è conservato il significante, per esempio il Mastro Ciliegia è rappresentato da un tocco, attrezzi e ciliegie; a loro volta i nomi dal significato imprecisato sono stati tradotti metaforicamente: Pinocchio -

${ }^{6}$ I principi dell'elaborazione del dizionario e della grammatica dell'emojitaliano nonché gli esempi sono stati attinti dal testo di Francesca Chiusaroli (2017, pp. 5-18). 
una figura che corre ${ }^{7}$, Geppetto (buon padre) - volto di uomo e un cuore, Grillo - un tocco e una trombetta. Si è rinunciato alla traduzione delle espressioni idiomatiche (ivi, pp. 10-12).

Nella traduzione è stata molto semplificata la grammatica italiana. L'emojitaliano è nato sulla base di analisi comparative delle lingue naturali (l'uso dell'ordine più comune della frase), i creatori si sono altrettanto ispirati ai ben conosciuti universali linguistici e anche ai progetti di lingue artificiali. L'emojitaliano è caratterizzato dalla prevalenza della paratassi sulla ipotassi; dall'ordine stabile della frase SVO (soggetto verbo - oggetto); la mancanza della flessione è controbilanciata dalla presenza di forme analitiche. I traduttori hanno cercato di mantenere le più importanti categorie semantico-strutturali e grammaticali (per esempio la faccia di bambino apposta a un sostantivo indica diminutivo, un segno ripetuto due volte indica plurale, un segno diacritico precede un verbo, le indicazioni per mezzo di frecce esprimono i tempi passato e futuro ecc.) (ivi, pp. 12-14).

Questa traduzione è nata in seguito al lavoro di molti individui che sceglievano dal repertorio a loro disponibile di segni emoji e combinavano quei segni sulla base del principio di collage-remix. Sembra chiaro che questo prodotto debba essere annoverato nel tipo di traduzione intersemiotica (da un codice verbale verso un altro sistema semiotico; Jakobson, 1959, p. 233), il lavoro del traduttore invece può essere paragonato all'attività del bricoleur (Lévi-Strauss, 1962, p. 27). Tale paragone, del resto, non è una novità nella terminologia traduttologica (cf. Majdzik, 2009).

Il bricoleur - sintetizziamo a grandi linee la concezione antropologica di Lévi-Strauss - esprime un tipo di atteggiamento nei confronti del mondo circostante che consiste nella creazione di cose nuove a partire dall'uso di materiali già disponibili, non sempre prodotti in vista della creazione di quelle cose specifiche. Quest'attività è facilmente riscon-

7 Pinocchio è anche una giovane pianta (simbolo di immaturità) o burattino (simbolo di macchina, quindi di tutto ciò che non è umano). Le motivazioni delle scelte di questi simboli sono presentate da Chiusaroli (ivi, pp. 5-18); la formulazione di interpretazioni anche in Majdzik (2018). 
trabile nell'esperienza di qualsiasi parlante di una lingua (il messaggio è la conseguenza di una scelta nel ventaglio delle possibilità del sistema linguistico), ma anche nell'esperienza di lavoro del traduttore-bricoleur:

I suoi strumenti e materiali sono rappresentati dai mezzi della lingua d'arrivo, i significati sono connotati dall'originale e dalla sua forma linguistica, dall'insieme dei codici letterari della lingua di partenza e dalla cultura della traduzione [...]. Il modo di maneggiare questi elementi dipende in grande misura dalle esperienze di vita del traduttore-bricoleur, dalla sua biografia, cultura letteraria, idioletto. (Majdzik, 2009, p. 158)

Sicuramente succede che il traduttore si basi non esclusivamente sulla materia della lingua d'arrivo, ma che la combini con elementi estranei ovvero non appartententi al sistema linguistico d'arrivo (ricorrendo all'equivalenza indiretta o semplicemente mantenendo la dominante oppure il punto di accesso al senso - come è spesso concepita l'equivalenza globale in varie teorie della traduzione - si allontana dai significati primari dell'originale o dagli equivalenti diretti per esempio lessicali). Senza dubbio tale bricolage traduttivo è più visibile là dove il traduttore ha fatto ricorso alla citazione, al prestito dall'originale o al cliché. Tutto ciò avvicina la traduzione alla citazione, il che è stato a suo tempo notato da Edward Balcerzan (2011, p. 173; trad. mia):

Dal punto di vista lessicale la citazione è il contrario della traduzione. Citando riferiamo letteralmente l'idea di qualcuno, traducendo ne produciamo una riformulazione. Tuttavia, "traduzione" e "citazione" si situano nello stesso campo retorico, rappresentano la stessa poetica che abbiamo chiamato "poetica della reminiscenza".

La citazione, l'allusione, il cliché letterario sono probabilmente le strategie intertestuali più riconoscibili proprie del traduttore-bricoleur. Tamara Brzostowska-Tereszkiewicz (2014) elenca accanto ad esse (o anche le include tra le altre strategie traduttive di retroguardia ${ }^{8}$ ) il collage, il ready-made e il centone.

8 A proposito di non originalità e retroguardia nelle pratiche poetiche contemporanee cf. Tabaszewska (2012). 
Le traduzioni-emoji (anche quelle che adoperano il pittogramma e la parola) possono essere senza dubbio incluse in quel tipo di tecniche traduttive. Originariamente, le traduzioni-emoji sono nate nell'ambito elettronico (il che non è contraddetto dal fatto che molte di esse, anche Pinocchio, siano state pubblicate in forma cartacea) e rappresentano un esempio di traduzione intersemiotica. Ewa Szczęsna (2018, p. 333) indica tre tipi di traduzione digitale: digitalizzazione (traduzione mediale; le opere acquisiscono una cornice ergodica), traduzione ipertestuale (sia la traduzione dell'ipertesto letterario sia la traduzione ipertestuale propriamente detta) e traduzione intersemiotica digitale (le traduzioni della letteratura tradizionale in forme digitali multisegniche, animazioni). La traduzione in emoji andrebbe assegnata al terzo tipo. La studiosa sottolinea il carattere creativo dell'atto traduttivo digitale, ciò situa questo fenomeno in prossimità di altre forme digitali dell'espressione (artistica), come la internet art o la poesia digitale (ciberpoesia):

La traduzione digitale è una trasformazione multidimensionale e creativa. Il carattere trasformativo della traduzione digitale si manifesta su vari piani: semiotico, tecnologico, culturale, dell'invenzione e dell'intenzione del traduttore. Il messaggio ottenuto in seguito a una tale traduzione è una rappresentazione del testo di partenza. La rappresentazione si estende dalle interpretazioni imitative in cerca di analogie attraverso le reinterpretazioni coscienti e procedure metatraduttive fino alle libere parafrasi, ai giochi intenzionali con l'originale. Tutte le forme della traduzione digitale sono adattamenti. (Szczęsna, 2018, p. 16; trad. mia)

La traduzione digitale comprende quelle forme di trasformazione del testo nelle quali la partecipazione delle tecnologie elettroniche ha un carattere creativo. Tuttavia, il Pinocchio italiano è un caso limite. E non perché alla fine sia stato stampato, il che l'ha strappato dall'ambiente elettronico primario. Le traduzioni digitali intersemiotiche esemplari si basano sulla trasformazione del testo grazie ai software creativi, agli strumenti creati per l'elaborazione tecnologica del testo. Il lavoro sulle traduzioni emoji, invece, consiste nelle scelte compiute da un soggetto umano (quindi non un algoritmo preprogrammato). Anche se dispone di un numero limitato di segni, infatti "ogni utente [...] di Internet $[\ldots]$ 
ha a disposizione (della scelta) solo l'insieme delle possibilità previsto precedentemente dai programmatori-autori” (ivi, p. 17).

Un contesto più ampio del fenomeno della emoji-traduzione sicuramente è rappresentato dalla traduzione sperimentale, o anche la cosiddetta post-traduzione (post-translation; cf. Gentzler, 2017; Brzostowska-Tereszkiewicz, 2018 ${ }^{9}$.

\section{TRADUZIONE E POETICA DEL COPY/PASTE}

Il concetto di remix così spesso usato per caratterizzare la cultura contemporanea, permette non solo di descrivere una grande parte di fenomeni culturali, ma anche di ridefinire le categorie umanistiche basilari come l'autore e il destinatario, l'artefatto, di descrivere le creazioni/ opere senza la fuga verso le valorizzazioni moderniste (differenziazioni di cultura alta e bassa), di descrivere la dialettica dell'originalità e della ripetizione o la circolazione dei testi ai tempi dei mezzi elettronici (cf. Gulik, Kaucz \& Onak, 2011, pp. 7-9). Il remix inizialmente era legato alla musica (il termine è significativo anche nel contesto dei diritti d'autore; cf. Lessig, 2009), ma poi si è velocemente allargato al resto delle arti (inclusi le arti visive, il teatro) e l'idea di mischiare, collegare, copiare e incollare (copy/paste), di riprodurre - come ho cercato di indicare anche più sopra - non è estranea nemmeno alla riflessione sulla traduzione.

Tomasz Plata, divulgatore del remix nella pratica teatrale, definisce il remix come segue:

È quel tipo di lavoro artistico che entra in un dialogo significativo con il lavoro di qualcun altro, un lavoro precedente: lo rifà, lo integra, lo sviluppa

9 Parlando dell'arte traduttiva contemporanea, Brzostowska-Tereszkiewicz afferma che essa presenti "una serie di mutazioni dall'illusione mimetica attraverso la dissoluzione delle illusioni nel paradigma avanguardistico della traduzione alle traduzionicentoni, alle installazioni metatraduttive astratte, alle metapoesie-oggetti che (almeno nella diagnosi di alcuni studiosi) suggellano la svolta verso il postmodernismo nella pratica traduttiva" (2018, p. 83). 
con l'intenzione di ridare vita, di rendere omaggio, di arrivare ai temi precedentemente nascosti, a volte di contestare. (Świątkowska, 2015)

Egli quindi richiama l'attenzione non solamente sul meccanismo stesso della creazione - sul remixare - quanto sulla ricezione dell'opera (originaria) e sulla sua reinterpretazione attraverso il procedimento del remix (e perciò anche sulla ricontestualizzazione). Il remix, soprattutto quello fatto con l'ausilio dei mezzi elettronici, ha infatti anzitutto un potenziale sul piano critico-ricettivo e ci dice tanto non solo dell'originale, ma anche del suo destinatario. Ewa Wójtowicz nota che "il concetto di remix [...] ha iniziato a funzionare nelle arti visive dal momento dell'entrata in quell'ambito della digitalizzazione" (2011, p. 12), benché pure i protoremix - fotomontaggi dei dadaisti - avessero il carattere dell'operare cosciente sui materiali di partenza nonché la carica critica tipica del remix (Nacher, Gulik \& Kaucz, 2011, p. 12). Oggi, innanzitutto nel contesto della creatività e della traduzione emoji, è difficile parlare di remix prescindendo dal mondo digitale; del resto la tecnologia fa ormai parte dell'esperienza culturale e sociale quotidiana. Ciò si traduce anche (nel modo più semplice) in disponibilità di strumenti elettronici che servono alla creazione di opere artistiche (tra queste la traduzione) e porta alla loro diffusione e democratizzazione ${ }^{10}$, le quali spesso vanno mano nella mano con l'anonimato dei creatori. Nel caso della già citata traduzione di Pinocchio, al progetto hanno preso parte tanti partecipanti che sono rimasti anonimi (la traduzione è un'opera collettiva). Una cosa simile è successa nel caso dell'emoji-traduzione di Moby Dick (ovvero il romanzo Emoji Dick) sorta con la partecipazione di Amazon Mechanical Turk $^{11}$. Parimenti, numerosi rebus, rifacimenti, traduzioni, "sciarade" ed

10 Alcune opere emoji nascono con un chiaro (spesso unico) intento scherzoso (il che del resto è promosso dalla stessa forma convenzionale dell'immagine emoji), più raramente parodico. Esistono spesso (o forse di solito) nell'area periferica dell'arte essendo frequentemente solo meme, battute. Del resto, è tipico delle creazioni artistiche elettroniche che si servono della tecnica del remix: "l'arte fornisce degli esempi che confermano la diffusione dell'estetica del remix, spesso al margine della cultura popolare, da qui il suo talvolta discutibile status" (Wójtowicz, 2011, p. 28; trad. mia).

11 Nel 2010 Fred Benenson ha pubblicato la traduzione in codice emoji del classico di Herman Melville. Emoji Dick è sorto come risultato del lavoro di molti creatori 
emoji-poesie (emojipoems) sono pubblicati in modo anonimo sulla rete. Però non è una regola ${ }^{12}$.

Opere e traduzioni con l'utilizzo di emoji si basano sul meccanismo del remix non esclusivamente per quel che riguarda l'attività di combinazione di segni al fine di costruire qualcosa di nuovo o di mischiare la lingua verbale e il codice iconico, ma anche per quel che riguarda l'uso dei segni creati al fine di comunicare tramite certi canali (quindi, una comunicazione tramite mezzi digitali, che avviene su dispositivi di comunicazione, piattaforme in rete e applicazioni mobili) con lo scopo di espressione artistica o di comunicazione al di fuori dei luoghi originariamente adibiti a tal fine, il che avvicina questi messaggi al fenomeno mash-up ${ }^{13}$ (un fenomeno simile al remix che indica "la combinazione di dati o funzionalità di due o più fonti esterne diverse per creare un servizio nuovo"; Sonvilla-Weiss, 2010, p. 8; cf. Nacher, Gulik \& Kaucz, 2011, p. 9). In molti casi in cui è coinvolto l'utilizzo degli emoji si arriva al trasferimento dell'ambito di funzionamento del testo dalla sfera elettronica al mondo non virtuale (per esempio allo spazio museale o alla circolazione cartacea). Allo stesso tempo vediamo come si diffonde l'uso - anche artistico - dei segni emoji il cui senso non si esaurisce solamente nella semplice riproduzione (nell'operazione copy-paste) come modo di esecuzione dell'atto creativo (dove il creatore diventa postproduttore; cf. Wójtowicz, 2011), ma soprattutto come una ricontestualizzazione e reinterpretazione significative. Queste ultime a loro volta indicano i meccanismi di funzionamento della traduzione in senso lato.

Adesso vorrei presentare qualche esempio di utilizzo degli emoji nella traduzione inter- e intrasemiotica, in particolare nell'arte concettuale che si serve degli emoji (in cui il problema della traduzione è presente

anonimi sulla piattaforma di crowdsourcing MTurk. Durante i lavori sulla traduzione si traducevano prima i vari frammenti di Moby Dick e poi per mezzo di una votazione si sceglievano le soluzioni migliori.

12 Sulla soggettività dei creatori di emoji-traduzioni nella prospettiva della svolta performativa cf. Majdzik (2018).

13 Aggiungiamo che su internet ci sono siti che offrono la possibilità di creare una propria opera emoji utilizzando gli emoji disponibili (per esempio emoji-maker.com, emojiart.org). 
implicitamente o esplicitamente). Roman Jakobson nel testo, fondamentale per la teoria della traduzione, On linguistic aspects of translation (1959) individua tre tipi di traduzione: a) traduzione endolinguistica (ovvero riformulazione, cioè l'interpretazione dei segni linguistici per mezzo di altri segni linguistici della stessa lingua), b) traduzione interlinguistica (ovvero traduzione propriamente detta, cioè l'interpretazione dei segni linguistici per mezzo dei segni di un'altra lingua), c) traduzione intersemiotica (ovvero trasmutazione, cioè l'interpretazione dei segni linguistici per mezzo di sistemi di segni non linguistici (ivi, p. 233). Edward Balcerzan invece aggiunge a questa triade classica anche la traduzione intrasemiotica, cioè il passaggio da un sistema non linguistico a un altro sistema non linguistico (2011, pp. 301-303). L'anonima opera Ceci n'est pas une pipe da me menzionata all'inizio è un esempio di traduzione intrasemiotica (dalla pittura su tela verso la lingua delle icone emoji) che tematizza la traduzione intersemiotica (delle parole in immagini).

Tra le emoji-opere troviamo rifacimenti, ecfrasi, traduzioni intersemiotiche, indovinelli, sciarade, rebus. Osserviamo meglio alcuni esempi: l'emojipoem che presenta la testa di un uccello grigio, una donna con la corona, un aereo in volo e un paesaggio serale. L'opera è accompagnata dal titolo The owl of Minerva takes flight when the shades of night are gathering, questo ripreso dagli Scritti di filosofia del diritto hegeliani (autore Blake, data: 2012, 23 dicembre; vedi Fig. 1a). Un'altra opera composta dalla figura di un fantasma e dalla testa con il balloon da fumetto che suggerisce la meditazione è stata intitolata Hamlet (anonimo; 2012, 24 dicembre; Fig. 1b). Un'altra ancora con l'ausilio dei segni emoji ricrea la scena della creazione di Adamo ed è intitolata After $\mathrm{Mi}$ chelangelo (anonimo; 2012, 24 dicembre; Fig. 1d). Riconosciamo qui ovviamente classici della filosofia, opere pittoriche, letterarie e anche cinematografiche. Numerose opere emoji si richiamano a cliché, a simboli della cultura popolare, a stereotipi ecc. Possono essere scherzose, ironiche, rivelatorie, ma anche volgari. Come per esempio America II firmato da R.A.J. (2012, 20 dicembre; Fig. 1e) che ricrea la composizione grafica della bandiera degli Stati Uniti per mezzo di illustrazioni di hamburger, fette biscottate, patatine e posate o la serie di immagini Sext che allude all'atto sessuale (anonimo; 2012, 8 ottobre; Fig. 1c). 
a)

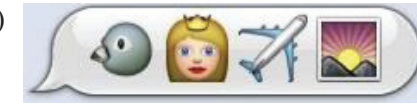

b)

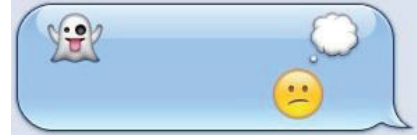

c)

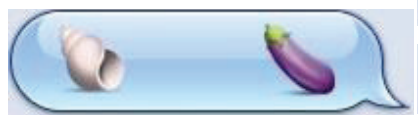

d)

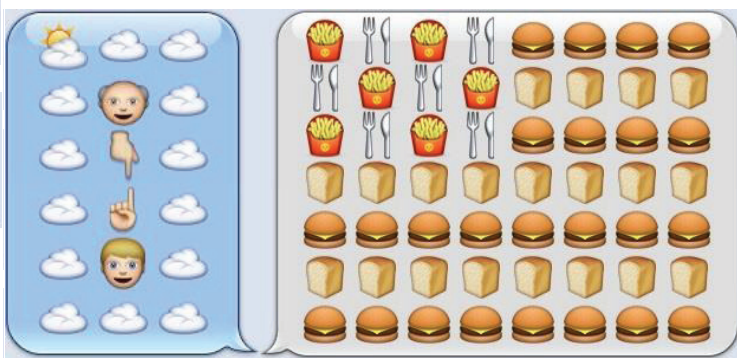

FigURA 1. Le emojipoem scelte dal portale emojipoems.tumblr.com

La creatività di questo tipo, anche se nasce come scherzo, si richiama spesso all'arte elitaria, alta o a contenuti seri e il suo umorismo è il risultato proprio di una tale giustapposizione contrastiva di contenuti seri e immagini triviali. Di qui la nascita in rete di frequenti concorsi per la creazione di traduzioni emoji (per esempio la trascrizione dei titoli di film per mezzo degli emoji) o per indovinare gli originali dei testi emoji (per esempio le traduzioni intersemiotiche dei classici della letteratura, le poesie di Emily Dickinson [il progetto Emoji Dickinson su Twitter], William Blake, Walt Whitman, Philip Larkin [Spiegelman \& Rankin-Gee, 2017]). Inoltre, si possono incontrare rebus, anche politici, che ricordano le realizzazioni classiche di quel tipo di giochi (per esempio un discorso di Barack Obama illustrato con emoji, 2015). Le realizzazioni qui menzionate di opere emoji sono nate ed esistono nella rete internet. Chiaramente, entrano nella circolazione comunicativa, ma sembra che quel che di fatto permette di legittimare la creatività emoji sia l'entrata nella circolazione non elettronica o una connessione più univoca con la creatività artistica. Per questo molti creatori cercano una realizzazione più concreta delle opere emoji. In questo caso entra in gioco la pubblicazione cartacea: in forma di libro, di poster o dipinto. Nell'arte concettuale digitale (anche nell'arte della traduzione) in tale modo viene portata a termine l'opera la cui stampa (o altra forma di pubblicazione) rappresenta il coronamento dello sforzo profuso nel progetto $^{14}$. Una cosa simile è successa con il progetto Emoji Dick (Be-

${ }^{14} \mathrm{Ci}$ servano d'esempio i progetti della Corporazione Ha! art, per esempio la traduzione per mezzo di Google Translate di Ubu re di Alfred Jarry (2015) o la stampa 
nenson, 2010) e con la traduzione molto più sistematica di Pinocchio (di cui si è parlato sopra). Bisogna ricordare che nel caso dell'opera di Collodi abbiamo a che fare con una vera e propria serie traduttiva verso il sistema dei segni emoji. Questa serie è stata iniziata dal poster di Joe Hale intitolato Pleasureland (2015) che, però, è una emoji-traduzione molto poco codificata (l'autore restituisce la semantica delle parole senza preoccuparsi della grammatica). Hale ha creato un'intera serie di poster-traduzioni dei classici della letteratura per bambini, riproducendo tra gli altri Wonderland (2014): la traduzione di Alice nel Paese delle Meraviglie di Lewis Carroll o Neverland (2015): Le avventure di Peter Pan di James Matthew Barrie. Hale asserisce che l'enigmaticità e i nonsense di Alice nel Paese delle Meraviglie quasi impongono la loro traduzione per mezzo degli emoji perché essi creano "un effetto visivo che restituisce la natura assurda dell'originale" (Mufson, 2015).

La traduzione intersemiotica può avvenire anche con la direzionalità opposta, ovvero dai segni emoji al sistema verbale. Qui ovviamente non penso alle "traduzioni" che in un certo senso possono essere fatte di ogni emoji (come per esempio la Faccina sorridente), ma di fatto a una tipica traduzione di poesie in immagini verso una lingua naturale. Nel 2014 è stato pubblicato il risultato della cooperazione di due poetesse americane - Carina Finn e Stephanie Berger -, la raccolta The Grey Bird. La prima scriveva emoji-poesie (insiemi di immagini significanti in cui si ripeteva ostinatamente il motivo dell'uccello grigio), invece la seconda le traduceva in lingua inglese. Il risultato è una piccola raccolta poetica composta da 13 opere "doppie" (Finn \& Berger, 2014; cf. Kim, 2015). Sulla copertina si vedono i nomi di entrambe le artiste, ma la prima è indicata come autrice, la seconda come traduttrice (anche se

delle commissionate dall'autore del progetto Poesie a cento dollari fatta da Amazon Mechanical Turks (Marecki, 2015). Così come la lingua (come succede soprattutto nel caso delle lingue minori) è legittimata dall'esistenza al suo interno di traduzioni delle opere più rilevanti per una data cultura, così anche la emoji-traduzione è legittimata grazie al rango dell'opera (originale) che è tradotta verso il sistema emoji. Detto ciò, questa tesi generale può anche essere usata in modo scherzoso e sovversivo. Il progetto della traduzione della Bibbia in emoji dovrebbe essere indirizzato alla generazione dei "millennial" che altrimenti non capirebbero le Sacre Scritture (cf. Cuthbertson, 2016). 
non si può negare l'aspetto creativo del lavoro di Berger). Così è stato innalzato lo status dell'opera in emoji (di solito deprezzato in confronto all'arte della parola). Il libro è allo stesso tempo un commento riguardante il carattere creativo del lavoro del traduttore e il suo posto sulla mappa letteraria. L'utilizzo dei segni emoji è descritto anche dalla poesia verbale che solleva questo tema senza però adoperare le icone dell'Unicode:

The one that winks,

The one in hysterics,

The beer,

The wine,

The OK sign.

The shocked one,

The facepalm one,

The angel baby,

The thumbs up,

And the one throwing up.

Life can't be bad:

My frequent emojis aren't sad.

(Eryri, 2018)

Queste poesie semplici scritte da autori amatoriali spesso contestano l'uso degli emoji riconoscendo in essi e nei contatti mediati dalle piattaforme digitali popolari una minaccia alla complessità e significatività della comunicazione intersoggettiva (le opere di Emmy, di Jeremy Rascon $)^{15}$.

L'utilizzo degli emoji per la critica della cultura contemporanea, della natura mediata elettronicamente della comunicazione, dell'estetica triviale, del consumismo avviene attraverso le arti plastiche. L'emoji è entrato nella galleria d'arte $M o M a$ nel 2016. Proprio allora sono stati presentati a New York gli emoji classici, ovvero il progetto originario

15 Vedi il portale hellopoetry.com/words/emojis. 
delle immagini di Shigetaka Kurita ${ }^{16}$. A sua volta John Baldessari, concettualista americano che si serve volentieri del collage, della giustapposizione, delle più varie forme e tecniche dell'espressione, ha creato un ciclo di quadri ad acrilico che rappresentano degli emoji ingranditi, apponendo loro iscrizioni, citazioni dai film in cui è difficile scorgere il collegamento con i segni emoji scelti (cf. Artnet News, 2017). Baldessari esplora il potenziale narrativo dei quadri e il potere associativo della lingua nei limiti dell'opera artistica. L'installazione artistica di Carla Gannis intitolata The Garden of Emoji Delights è un trittico, un collage digitale che reinterpreta per mezzo degli emoji il capolavoro di Hieronymus Bosch il Giardino delle delizie. Questo chiaro esempio di traduzione intrasemiotica è spiegato dall'artista nel modo seguente:

My work examines the narrativity of 21st century representational technologies and questions the hybrid nature of identity, where virtual and real embodiments of self diverge and intersect. I feel akin to past and contemporary artists and writers who uncannily deconstruct rigid notions of reality and perception. The extension of this sensibility with computer-based applications is only natural to me as a reflection upon the Digital Age in which we all coexist. (Gannis, NA)

Servendosi degli emoji l'artista ricontestualizza e reinterpreta anche l'opera di Bosch; presentandola in un'edizione nuova, contemporanea e digitale, dice al riguardo molto su lui stesso (in conformità ai presupposti della cultura del remix). L'opera di Bosch ritrova un nuovo significato, parzialmente e fino ad un certo punto coincidente con le intenzioni e con le interpretazioni classiche dell'originale:

By replacing religious vocabulary with secular and contemporary digital symbols, Gannis reconstructs the powerful iconography at the core of Bosch's landscape in The Garden of Earthly Delights. Her stunning, pop art 2.0 digital collage explores and critiques consumerism and modern society through the three emoji-fied realms of Eden, Hell, and Earth. (Palop, 2014)

16 La mostra Inbox: The Original Emoji, by Shigetaka Kurita (The Museum of Modern Art, 09/12/2016-12/03/2017); il portale Mobirank.pl ci informa di voler pubblicare un libro che cataloghi i 176 emoji di Shigetaka Kurita (vedi Majchrzyk, NA). 
La traduzione intrasemiotica si basa sulla ricontestualizzazione dell'originale collocandolo nello spazio-tempo della contemporaneità e nel campo digitale.

\section{CONCLUSIONI}

Le opere che si servono di emoji o che fanno riferimento a emoji (come le poesie che ne parlano, ma non ne fanno uso) funzionano in uno spazio digitale (Internet), essendo spesso anonime, ma ora entrano anche nelle gallerie, il che legittima la loro presenza nel mondo dell'arte dove diventano simbolo della contemporaneità e dei problemi odierni dell'arte stessa e della comunicazione (come p. es. il rapporto tra l'arte e il divertimento, tra l'originale e la copia, il consumismo, la natura e le modalità della comunicazione interpersonale, il ruolo dell'immagine nella comunicazione digitale, la definizione dell'arte e dell'atto creativo, il ruolo della ripetizione e della copia nell'arte/nella comunicazione, la soggettività nel mondo dei media digitali ecc.). La traduzione ha contribuito al riconoscimento delle emoji come sistema di segni rilevante, con cui il soggetto può esprimersi (addirittura ideale per la rappresentazione dei suoi problemi, tra cui la descrizione della sua partecipazione al mondo digitale). L'esperimento Pinocchio in emojitaliano ha dimostrato che sia possibile sostituire completamente la parola con l'immagine (anche se non è facile capire il testo della traduzione). Per riconoscere la condizione dell'uomo e dell'arte (compresa l'arte della traduzione), altrettanto importanti sono ulteriori tentativi di utilizzare le emoji nella traduzione e nel remix, come la traduzione dall'immagine emoji verso un codice verbale o la traduzione intersemiotica con le emoji (sia la traduzione in emoji - il trittico di Gannis - sia la traduzione da emoji verso un codice convenzionale delle arti figurative - le opere di Baldessari).

Come è chiaro, vi sono altre opere d'arte che si servono degli emoji, della tecnica del remix, della traduzione inter- e intrasemiotica (ad esempio le emoji-composizioni pop art di Natalya Nova). Sono senza dubbio il segno delle possibilità dell'espressione artistica che può essere data agli utenti - dilettanti e professionisti - dagli strumenti della 
comunicazione disponibili nel mondo digitale. A sua volta, la traduzione può diventare in questo caso tanto metodo creativo quanto argomento dell'espressione artistica, scientifica, filosofica e questa impressione è abilmente confermata dall'emoji-operetta Ceci n'est pas une pipe. In quanto tale, la traduzione diventa una specie di creatività sperimentale (concettuale).

\section{BIBLIOGRAFIA}

Artnet News (2017, Nov. 28). John Baldessari Has Transformed Emoji Into

New Icons of Conceptual Art. Retrieved from https://news.artnet.com/ art-world/john-baldessari-emoji-1161443.

Balcerzan, E. (2011). Ttumaczenie jako „wojna światów”. W kręgu translatologii i komparatystyki (3rd edition). Poznań: Wydawnictwo Naukowe UAM.

Benenson, F. (2010). Emoji Dick. Retrieved from emojidick.com.

Brzostowska-Tereszkiewicz, T. (2014). Kolaż, centon, ready-made jako techniki translatorskie. In P. Fast (Ed.), Strategie translatorskie od modernizmu do (post)- postmodernizmu (pp. 57-92). Katowice: Śląsk.

Brzostowska-Tereszkiewicz, T. (2018). Sztuka post-przekładu. Przekłady Literatur Słowiańskich, 9/1, 77-97.

Chiusaroli, F. (2017). Tradurre "Pinocchio" in "Emojitaliano". In F. Chiusaroli, J. Monti, \& F. Sangati (translation), Pinocchio in Emojitaliano (pp. 5-18). Sesto Fiorentino: Apice libri.

Chiusaroli, F., Monti, J., \& Sangati, F. (Eds. \& translation). (2017). Pinocchio in Emojitaliano. Sesto Fiorentino: Apice libri.

Cuthbertson, A. (2016, Feb. 6). Bible translated into emoji as 'Scripture 4 Millennials'. Retrieved from newsweek.com/emoji-bible-translationmillennials-465687.

Emojipoems (2012, Oct. 20). Ceci n'est pas une pipe. Retrieved from https:// emojipoems.tumblr.com/post/33977122922/ceci-nest-pas-une-pipe.

Eryri (2018, Nov.) Frequently Used Emojis. Retrieved from https://hellopoetry.com/words/emojis.

Finn, S., \& Berger, C. (2014). The Grey Bird: Thirteen Emoji Poems in Translation. [Place of publication not identified]: Coconut Books,

Foucault, M. (1973). Ceci n'est pas une pipe. Fontfroide-le-Haut: Fata Morgana. 
Gannis, C. (n.d.). Statement from the Artist. Retrieved from http://transfergallery.com/carla-gannis.

Gentzler, E. (2017). Translation and Rewriting in the Age of Post-Translation Studies. New York: Routledge.

Gulik, M., Kaucz, P., \& Onak, L. (Eds.). (2011). Remiks. Teorie i Praktyki. Kraków: Hub Wydawniczy Rozdzielczość Chleba.

Jakobson, R. (1959). On linguistic aspects of translation. In R.A. Brower (Ed.), On translation (pp. 232-239). Cambridge: Harvard University Press.

Jarry, A. (2015). Ubu Król, translated by A. Małecka, \& P. Marecki (with Google Translate). Kraków: ha!art.

Kalaga, W. (2010). Liberatura: słowo, ikona, przestrzeń [wstęp]. In Z. Fajfer, Liberatura czyli literatura totalna. Teksty zebrane z lat 1999-2009 (pp. 9-19). Kraków: ha!art.

Kim, G. (2015, Mar. 30). Emoji in Translation: A Review of The Grey Bird. Retrieved from weird-sister.com/2015/03/30/emoji-in-translation-a-review-of-the-grey-bird.

Lessig, L. (2009). Remiks. Aby sztuka i biznes rozkwitaty w hybrydowej gospodarce. Warszawa: Wydawnictwa Akademickie i Profesjonalne.

Lévi-Strauss, C. (1962). La Pensée sauvage. Paris: Plon.

Majdzik, K. (2009). Tłumacz-bricoleur (o polskim przekładzie „Ministerstwa Bólu" Dubravki Ugresić). In P. Fast, \& A. Świeściak (Eds.), Sztuka przekładu-interpretacje (pp. 157-174). Katowice: Śląsk.

Majdzik, K. (2018). Pinocchio in Emojitaliano. Przekład eksperymentalny w kulturze zwrotu wizualnego i performatywnego. Przekłady Literatur Stowiańskich, 9/1, 125-146.

Mitchell, W.J.T. (2012). Piśmienność wizualna czy wizualność piśmienna? Teksty Drugie, 1-2 (133-134), 153-163.

Majchrzyk, Ł. (n.d.). Kolekcja 176 oryginalnych Emoji w jednej książce. Retrieved from https://mobirank.pl/2018/05/01/kolekcja-176-oryginalnych-emoji-w-jednej-ksiazce.

Marecki, P. (2015). Wiersze za sto dolarów. Kraków: ha!art.

Mufson, B. (2015, Jan. 2). Author Translates All of ,Alice in Wonderland' into Emojis. Retrieved from https://www.vice.com/en_us/article/ez5vd4/author-translates-all-of-alice-in-wonderland-into-emojis.

Nacher, A., Gulik, M., \& Kaucz, P. (2011). Post-teorie i re -praktyki. Wprowadzenie do remiksu. In M. Gulik, P. Kaucz, \& L. Onak (Eds.), Remiks. Teorie i Praktyki (pp. 7-15). Kraków: Hub Wydawniczy Rozdzielczość Chleba. 
Obama, B. (2015, Jan. 21). State of the Union... in emojis. Retrieved from theguardian.com/us-news/ng-interactive/2015/jan/20/-sp-state-of-theunion-2015-address-obama-emoji.

Palop, B. (2014, Oct. 25). Step Inside 'The Garden of Emoji Delights'. Retrieved from https://www.vice.com/en_us/article/53w39a/step-insidethe-garden-of-emoji-delights.

Prisco, J. (2018, May 23). Shigetaka Kurita: The man who invented emoji. Retrieved from https://edition.cnn.com/style/article/emojishigetaka-kurita-standards-manual/index.html.

Sonvilla-Weiss, S. (Ed.). (2010). Mashup Cultures. Wien, New York: Springer. Spiegelman, N., \& Rankin-Gee, R. (2017, Aug. 18). Emoji Poetry Contest. Retrieved from theparisreview.org/blog/2017/08/18/emoji-poetry.

Świątkowska, W. (2015). Remiks [hasło]. Elektroniczna Encyklopedia Teatru Polskiego. Retrieved from http://www.encyklopediateatru.pl/hasla/351/ remiks.

Tabaszewska, J. (2012). Nieoryginalna ariergarda. Koncepcja Marjorie Perloff jako próba diagnozy statusu współczesnej poezji. Teksty Drugie, 3, 197-213.

Tokarz, B. (1998). Wzorzec, podobieństwo, przypominanie. Katowice: Śląsk. Tokarz, B. (2010). Spotkania: czasoprzestrzeń przekładu artystycznego. Katowice: Wydawnictwo Uniwersytetu Śląskiego.

Szczęsna, E. (2018). Cyfrowa semiopoetyka. Warszawa: Instytut Badań Literackich $\mathrm{PAN}$.

Wójtowicz, E. (2011). Twórca jako postproducent - między postmedialnym remiksem a reprogramowaniem kultury. In M. Gulik, P. Kaucz, \& L. Onak (Eds.), Remiks. Teorie i Praktyki (pp. 16-30). Kraków: Hub Wydawniczy Rozdzielczość Chleba.

Riassunto: L'articolo presenta alcune forme di traduzione intersemiotica e intrasemiotica con l'utilizzo degli emoji, cioè dei segni iconici adoperati nella comunicazione elettronica dagli utenti di applicazioni e di piattaforme web diffuse (come p. es. Twitter, Facebook, Messenger). L'uso degli emoji è sempre più comune, ciò è confermato dalla quantità costantemente più alta di messaggi nonché di opere d'arte composti esclusivamente da tali segni. Sempre più spesso si cerca di trasporre anche testi verbali più ampi (tra questi opere letterarie) al sistema non verbale dei segni emoji. Il coronamento di tale lavoro è rappresentato dalla traduzione sperimentale di Avventure di Pinocchio. Storia di un burattino di Carlo Collodi, un classico della letteratura per bambini, dalla lingua italiana all'emojitaliano, lingua codificata dai partecipanti all'esperimento (la traduzione è stata fatta sulla piattaforma Twitter e nel 2017 è uscita la sua versione cartacea). Le opere d'arte che fanno uso di emoji compaiono anche nei musei. Il trittico The Garden of Emoji Delights di Carla Gannis è un bell' 
esempio di traduzione intrasemiotica (da un codice non verbale a un altro codice non verbale) della famosa opera di Hieronymus Bosch. Un uso simile degli emoji è legato ai cambiamenti paradigmatici delle materie umanistiche, connessi alle varie svolte: digitali, iconica, performativa nonché alla cultura del remix, attualmente molto diffusa.

Parole chiave: emoji, emojitaliano, traduzione sperimentale, traduzione digitale, cultura del remix. 